\title{
Análisis de un protocolo preventivo contextualizado en jóvenes futbolistas
}

Analysis of a proposed preventive program for youth football players

Rafael Lorente-Corvi'

1. Preparador físico de El Mar Menor Fútbol Club.

\begin{abstract}
Resumen
El fútbol es un deporte altamente lesivo debido a diversos factores. La lesión disminuye el rendimiento no solo del jugador sino también del equipo, lo que crea la necesidad de establecer programas preventivos para paliar, en la medida de lo posible, la tasa lesional. El objetivo del presente estudio fue diseñar un programa preventivo contextualizado y analizar la reducción lesional derivada de su implementación en jóvenes futbolistas. La muestra estuvo compuesta por 20 futbolistas de la categoría juvenil del Fútbol Club Cartagena, con una media de 6 horas de entrenamiento semanal. Tras una breve valoración inicial, llevaron a cabo un nuevo protocolo de prevención con diferentes ejercicios agrupados en 2 niveles de dificultad. Se realizó un análisis descriptivo de cada una de las variables cuantitativas. Los resultados muestran una disminución del $65 \%$ del número total lesiones, entre temporadas, y en gran medida de las graves. La tasa de reducción lesiva obtenida está por encima de diferentes estudios que analizan la eficacia en diversos programas preventivos. Un programa de entrenamiento preventivo basado en la contextualización de los participantes ha resultado en una reducción de la tasa lesiva. Los entrenadores y profesionales del deporte disponen de una herramienta para disminuir el riesgo de lesión.
\end{abstract}

Palabras clave: Prevención, fútbol juvenil, lesión, ratio de lesión.

\begin{abstract}
Football is a highly injury-prone sport due to several factors. Injuries diminish the performance not only of the player but also of the whole team, which creates the need to establish preventives programs to alleviate the injury rate as much as possible. The objective of this research was to design a contextualized preventive program and analyze the injury reduction derived from the implementation in young footballers. The sample consisted of 20 footballers from the under-19 category of Fútbol Club Cartagena, with an average of 6 hours of training per week. After a brief initial assessment, they carried out a new prevention protocol with different exercises grouped into two levels of difficulty. A descriptive analysis was performed for each of the quantitative variables. The results show a decrease of $65 \%$ in the total number of injuries between seasons, and to a great extent to those more severe. The injury reduction rate is above different studies that analyze the effectiveness of different preventive programs. A preventive training program based on the contextualization of the participants has resulted in a reduction of the injury rate. Coaches and sport professionals have a tool to reduce the risk of injury.
\end{abstract}

Keywords: Prevention, sub-18 soccer, injury, injury ratio.

Recibido: 13 de julio de 2021

Aceptado: 18 de agosto de 2021

Publicado: 01 de diciembre de 2021

Como citar (APA): Lorente-Corvi, R. (2021). Análisis de un protocolo preventivo contextualizado en jóvenes futbolistas. JUMP, (4), 10-25. https://doi.org/10.17561/jump.n4.2

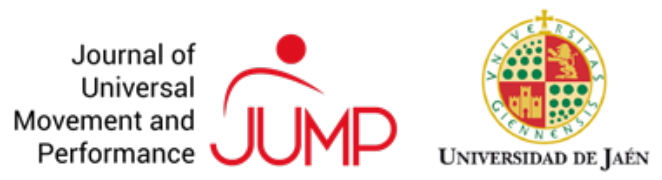




\section{Introducción}

El fútbol es uno de los deportes más populares en todo el mundo (Mufty et al., 2015). Su práctica no conoce fronteras pues según una encuesta realizada por la FIFA en 2006 hay aproximadamente 270 millones de personas involucradas activamente, lo que engloba a árbitros, técnicos y directivos, suponiendo en torno al $4 \%$ de la población mundial.

En España, hay más de 900.000 licencias federativas de fútbol según los datos publicados por el Consejo Superior de Deportes en 2016, suponiendo un aumento de más del $10 \%$ respecto a los valores establecidos en 2011. Este incremento en la práctica futbolística se ve reflejado también en un aumento de las lesiones sufridas por los jóvenes jugadores en etapas de formación, pues el deporte además de tener grandes beneficios sobre la salud, conlleva unas grandes exigencias físicas, técnicas, tácticas, psicológicas y fisiológicas (Slimani et al., 2017).

La literatura científica ha demostrado que el fútbol es un deporte con un alto riesgo lesional debido a la elevada participación (Acero \& Peñas, 2005; Brandt, 2017) y a la gran exigencia fisca requerida (saltos, aceleraciones, frenadas, etc.) (Cross et al., 2018; Drawer \& Fuller, 2002; Slimani et al., 2004). El concepto de lesión ha sido definido como todo fenómeno que ocurre en función de las horas de exposición durante las sesiones de entrenamiento o en competiciones y que supone una ausencia durante un entrenamiento o partido (Ekstrand, Waldén \& Hägglund, 2004). Las lesiones se deben principalmente a dos factores: a) intrínsecos, relacionados con los factores biológicos característicos de cada individuo como la edad, el sexo, la capacidad de producción de fuerza, la fatiga, la flexibilidad, los diferentes factores hormonales, la coordinación neuromuscular, la lateralidad, la alteración del gesto deportivo, la laxitud ligamentosa (asociada al ejercicio), el retraso electromecánico, la alteración propioceptiva y la reincidencia lesional; y b) extrínsecos, relacionados con todas aquellas variables externas como el terreno de juego, los equipamientos, los adversarios (por contacto) y las condiciones climáticas (Eirale, Gillogly, Singh \& Chamari, 2017; Llana-Belloch, PérezSoriano \& Lledó-Figueres, 2010; Orchard et al., 2013; Romiti, Finch \& Gabbe, 2007). De tal modo que las lesiones se analizan en función al número de lesiones sufridas y las horas de exposición.

Diversos autores han estudiado la relación existente entre las horas de exposición y el número de lesiones. Morgan \& Oberlander (2001) establecen una ratio de lesiones general de 35,3 por cada 1000 horas de exposición. También se encuentran valores próximos a 25 lesiones por 1000 horas de exposición en diferentes categorías (Arnason et al., 2004; Hägglund, Waldén \& Ekstrand, 2003; Waldén, Hägglund \& Ekstrand, 2005). Hootman, Dick \& Agel (2007) establecen una ratio de 18,8 por cada 1000 horas de exposición. Existen estudios que definen ratios lesionales menores pero que de igual modo acarrean una gran tasa de lesiones, Ekstrand et al. (2004) y Yoon, Chai \& Shin (2004) obtienen resultados en torno a 9 lesiones por cada 1000 horas de exposición e incluso 6,4 lesiones por cada 1000 horas de exposición (Pérez-Pemán \& Casajús-Mallén, 2013). Es por ello que nace la necesidad de reducir la tan elevada tasa de lesiones.

En los últimos años, las grandes entidades del mundo de la investigación deportiva y los profesionales del deporte y la salud han diseñado diversos protocolos para la reducción lesional. Cada uno aborda unos contenidos y diseña, por consiguiente, un programa con características diferentes.

Entre los protocolos más utilizados e investigados está el "Knee Injury Prevention Program" (KIPP) cuyo objetivo principal es la reducción del número de lesiones de LCA en jóvenes jugadores ya que el $36 \%$ de las lesiones sufridas se localizan en la articulación de la rodilla (LaBella et al., 2011; Larruskain, Lekue, Diaz, Odriozola \& Gil, 2018). El programa presenta una gran variedad de ejercicios con diferentes progresiones de carga, donde los contenidos principales son (1) carrera, (2) movilidad dinámica, (3) fortalecimiento, (4) pliometría y (5) agilidad. Se ha registrado una reducción de la ratio lesional de LCA entre grupo control y grupo experimental, aunque no fue estadísticamente significativa $(p=.09)$. 
Siguiendo con la línea de reducción de lesiones en la articulación de la rodilla en jóvenes jugadores de fútbol se creó el programa preventivo "Harmoknee" (Kiani, Hellquist, Ahlqvist, Gedeborg \& Byberg, 2010). Este programa de calentamiento ha experimentado una gran aceptación gracias a su sencillez, ausencia de material y eficacia en la reducción de lesiones en dicha articulación. Está formado por unos contenidos a realizar en cada sesión, (1) calentamiento, (2) activación muscular, (3) equilibrio, (4) fuerza y (5) estabilidad del CORE.

El programa neuromuscular Knäkontroll (Knäkontroll, SISU Idrottsböcker ${ }^{\odot}$, Sweden, 2005), tiene también como objetivo la reducción de lesiones agudas en la rodilla en jóvenes deportistas. El programa consta de seis ejercicios: (1) sentadilla unipodal, (2) puente lumbar dinámico, (3) sentadilla bipodal, (4) plancha isométrica frontal, (5) zancadas, y (6) técnicas de salto y caída. Cada ejercicio presenta una variante para realizar en parejas, así como una progresión de menor a mayor dificultad. En un estudio Ilevado a cabo por Waldén, Hägglund, Orchard, Kristenson \& Ekstrand (2013) se comprobó que dicho protocolo reduce significativamente la incidencia lesional de LCA.

Uno de los protocolos más efectivos en cuanto a la reducción de la incidencia lesional, es el programa preventivo "FIFA 11+" (RoblesPalazón \& Sainz de Baranda, 2017). Propuesto por la Fédération Internationale de Football Association (FIFA) y diseñado por el Centro Médico y de Investigación de la FIFA (F-MARC) junto con el Centro Deportivo Traumatológico y de Investigación de Oslo, publicado por Soligard et al. (2008). Este protocolo tiene por objetivo principal la prevención de lesiones del tren inferior de jóvenes jugadores de fútbol. El "FIFA 11+" es un programa multicomponente, que engloba ejercicios dinámicos centrados en los principales factores de riesgo intrínsecos que afectan a las lesiones del tren inferior. El protocolo se divide en tres apartados: (1) ejercicios de carrera, (2) fuerza, pliometría y equilibrio, y (3) ejercicios de carrera con transferencia al fútbol. Dentro de cada uno de los apartados, los ejercicios se subdividen en 3 niveles distintos en función del nivel del sujeto. Los creadores de dicho protocolo aconsejan la realización de este trabajo preventivo con una frecuencia mínima de dos veces a la semana durante al menos 3 meses consecutivos para obtener resultados (Owoeye, Akinbo, Tella, \& Olawale, 2014). Aunque se ha demostrado recientemente que con 4 semanas es suficiente para lograr el objetivo del protocolo (Ayala et al., 2017). Diferentes estudios han demostrado que una alta adherencia a dicho protocolo reduce notablemente la tasa lesional de los jugadores frente a aquellos que no realizaban el trabajo preventivo frecuentemente (Steffen et al., 2013). La facilidad de uso, el poco recurso temporal que precisa y la más que demostrada eficacia hacen de este protocolo una gran herramienta para los profesionales del deporte.

En los últimos 10 años han sido publicados diferentes trabajos sobre distintos programas preventivos. A pesar de ello, el número de lesiones no se han reducido notablemente e incluso en algunos casos se aprecia un aumento de la ratio lesional (Hammes et al., 2015; Van Beijsterveldt et al., 2012).

Por ello, el principal objetivo del presente trabajo ha sido diseñar un protocolo de prevención de lesiones contextualizado en un equipo de fútbol de categoría juvenil de fútbol. Un objetivo secundario fue analizar los efectos del programa preventivo contextualizado sobre la incidencia lesional.

\section{Material y Método}

\section{Muestra}

La muestra objeto de estudio estuvo compuesta por 20 jugadores de fútbol pertenecientes al Fútbol Club Cartagena en la categoría Nacional Juvenil durante las temporadas 2016/17 y 2017/18, donde realizaban 4 sesiones de entrenamiento semanales de una hora y media cada una. (Tabla 1). Antes de la recolección de datos, los participantes completaron un cuestionario, modificado de Fuller et al. (2006), que contenía preguntas sobre sus antecedentes deportivos (posición, extremidad dominante y experiencia deportiva); características antropométricas (edad, peso y talla); y aspectos relacionados con el entrenamiento (frecuencia de entrenamiento semanal y horas de entrenamiento semanal) (anexo 1). 
Los datos de los cuestionarios informaron que la muestra fue homogénea en posibles variables de confusión, tales como masa corporal, altura, edad, régimen de entrenamiento (de una competición y de 2-3 días de entrenamiento), condiciones climáticas, nivel competitivo, periodo de descanso y experiencia deportiva (al menos 6 años). Además, ninguno de los futbolistas había participado en un régimen sistemático y específico de entrenamiento de prevención de lesiones en los últimos 6 meses.

Tabla 1. Datos demográficos de los 20 jóvenes futbolistas.

\begin{tabular}{lccc}
\hline & Mínimo & Máximo & Total $(\mathrm{n}=20)$ \\
\hline Edad (años) & 16 & 18 & $17,1 \pm 0,9$ \\
Peso (kg) & 50 & 80,3 & $65,9 \pm 9$ \\
Talla $(\mathrm{cm})$ & 154 & 190 & $174,1 \pm 8,9$ \\
$\begin{array}{l}\text { Años de experiencia } \\
\text { Horas de entrenamiento }\end{array}$ & 7 & 10 & $8,6 \pm 1,3$ \\
$\begin{array}{l}\text { semanal } \\
\text { Frecuencia de }\end{array}$ & 6 & 6 & 6 \\
entrenamiento semanal & 4 & 4 & 4 \\
\hline
\end{tabular}

Los criterios de exclusión fueron: (1) no asistir a un mínimo de 3 sesiones semanales, (2) no disponer de ficha federativa en la categoría Nacional Juvenil tanto en la temporada 2016/17 y 2017/18, (3) no completar la ficha de registro de lesiones, y (4) no entregar la hoja de consentimiento informado firmada por los padres.

Tanto los futbolistas (padres o tutores de los futbolistas menores de edad) como cuerpo técnico del equipo y responsables del Club fueron verbalmente informados de la metodología a utilizar, así como de los propósitos y posibles riesgos del estudio, y cada uno de ellos firmó un consentimiento informado. El presente estudio pertenece a un proyecto de investigación aprobado por el Comité Ético y Científico de la Universidad de Murcia (España) (ID: 1551/2017).

\section{Procedimiento}

Previamente a la recogida de datos, se solicitó permiso al Club y al entrenador para poder llevar a cabo el estudio. Además, los padres o tutores fueron informados del propósito y procedimiento de la investigación y cumplimentaron un consentimiento informado.

Los participantes completaron un cuestionario referente a sus características antropométricas, su historial deportivo y lesional durante las primeras sesiones de las pretemporadas de los años 2016y 2017. Tras la recolección delos datos, se realizó una valoración inicial con diferentes pruebas para valorar cuantitativamente los factores de riesgo de lesión de los participantes.

Durante la temporada 2016-2017 únicamente se llevó a cabo un registro de las lesiones sufridas en los entrenamientos y partidos. Para la temporada 2017-2018 se implementó un protocolo de prevención de lesiones diseñado en función de los datos obtenidos en la valoración inicial y las características de la muestra. Durante esta temporada también se registraron las lesiones sufridas durante los entrenamientos y los partidos. Se realizó un periodo de familiarización de 2 semanas con los ejercicios utilizados para el protocolo, con el fin de corregir posibles errores en la técnica de ejecución de los diferentes ejercicios. Una vez finalizado este período, se llevó a cabo el programa establecido, realizando el nivel 1 de los ejercicios de fuerza durante la primera vuelta de la temporada y el nivel 2 en la segunda. La frecuencia de realización fue de 2 sesiones a la semana.

\section{Valoración inicial del riesgo de lesión}

Se realizó el primer día de ambas pretemporadas. El objetivo de la valoración inicial fue establecer el nivel físico de partida de los jugadores y analizar cuantitativamente los factores de riesgo de lesión de la muestra. Para evaluar la estabilidad dinámica del tren inferior, se utilizó el Y-Balance Test (Gribble et al., 2016), donde se valora la estabilidad en el plano anterior, posterolateral y posteromedial. La agilidad se evaluó mediante el test Illinois, dada su alta validación y reproductibilidad (Hachana et al., 2013; Negra et al., 2017; Roozen, 2004). Para medir el salto horizontal, se utilizó la batería de Single-Legged Hop Test, que consta de una serie de test funcionales que valoran la amplitud de salto frontal monopodal, los cambios de dirección, la aceleración y desaceleración, así como la estabilidad de la rodilla y posibles asimetrías (Logerstedt et al., 2012; Noyes, Barber \& Mangine, 1991; Reid, Birmingham, Stratford, Alcock \& Giffin, 2007). La batería de pruebas está formada por (1) Single Hop Test, (2) Triple Hop Test, (3) Crossover Hop Test y (4) Timed Hop Test. En cuanto al salto vertical, se valoró mediante la prueba Counter Movement Jump (CMJ), para la que se 
utilizó la app "MyJump 2", validada por Haynes, Bishop, Antrobus \& Brazier (2018).

\section{Protocolo}

El diseño del protocolo está basado en los contenidos comunes de los programas publicados por las diferentes instituciones deportivas de gran prestigio en el ámbito de la prevención de lesiones. El protocolo (tabla 2) contiene diferentes bloques de contenido: (1) carrera, (2) movilidad de cadera, (3) estiramientos dinámicos, (4A) ejercicios de fuerza - nivel 1, y (4B) ejercicios de fuerza - nivel 2. La frecuencia semanal fue de 2 entrenamientos semanales (anexo 2).

Los ejercicios seleccionados tratan de satisfacer las múltiples y diferentes demandas que exige el fútbol, de tal modo que presenta ejercicios para diferentes musculaturas y articulaciones. Los criterios utilizados para la división de los ejercicios de fuerza en 2 niveles son: la duración de los ejercicios, el número de series y de repeticiones, el número de apoyos, la activación de la musculatura y el grado de activación neuromuscular.

El primer bloque de contenidos del protocolo es la carrera, realizándose un bloque de 2' a una intensidad moderada, buscando la activación muscular y cardiorrespiratoria. Seguidamente, en el segundo apartado, se realizarán ejercicios que mejoren la movilidad de la cadera, vital para que el rango de movimiento (ROM) sea adecuado y evitar así posibles lesiones inducidas por un déficit en él. Los ejercicios seleccionados trabajan todos los posibles movimientos que la cadera puede realizar de forma dinámica (flexión, extensión, abducción, aducción y rotación externa e interna) (Fousekis, Tsepis, Poulmedis, Athanasopoulos \& Vagenas, 2011; Mahieu et al., 2007; Taunton et al., 2002).

Posteriormente, en el tercer apartado, se realizan una serie de estiramientos dinámicos, los cuales mejoran en gran medida el rango de movimiento, el ciclo estiramientoacortamiento y aumentan la temperatura corporal (Ayala \& Sainz de Baranda, 2010). Los ejercicios seleccionados se centran principalmente en la musculatura del tren inferior dadas las exigencias del deporte en cuestión. Siguiendo un orden descendente, que va de la musculatura de la cintura pélvica hasta la musculatura de los tobillos. Los ejercicios engloban algunas de las recomendaciones de Boyle (2017). Toda la serie de estiramientos dinámicos se realiza en un recorrido de 10 metros, donde el jugador avanza en línea recta hasta completar la distancia y vuelve a la posición inicial andando o trotando para realizar el siguiente ejercicio.

El cuarto bloque está compuesto por los ejercicios de fuerza diferenciando dos niveles. El primer nivel de dificultad está compuesto por 6 ejercicios: Sentadilla frontal (2x12-15 reps), Hip Thrust (2x6-14 reps), Nordic Hamstring (2x4-6 reps), Copenhagen ( $2 \times 8$ cada pierna), Zancadas frontales con extensión de tobillo (1x10 cada pierna) y Plancha Isométrica Frontal (6x10seg / d 2seg).

El primer ejercicio es la sentadilla bipodal frontal, destinado a la musculatura de la rodilla, ésta presenta una mayor activación del cuádriceps respecto a la sentadilla tradicional como demuestran los resultados de la electromiografía (EMG) de diferentes estudios (Boeckh-Behrens \& Buskies, 2005; Bompa, 2000; Contreras, 2010a). En este caso, se utilizó como resistencia una goma elástica. Se realizaron 2 series de 12 a 15 repeticiones.

La musculatura glútea es una de las más solicitadas y determinantes para el buen rendimiento del futbolista (Stefanyshyn \& Nigg, 1998). El "Hip Thrust", es un ejercicio destinado al fortalecimiento de dicha musculatura mediante un impulso horizontal (anteroposterior). Según un estudio de Contreras (2009b), el "hip thrust" es uno de los ejercicios que mayor activación glútea e isquiosural produce, medido con EMG. El impulso horizontal llega a activar la musculatura glútea tres veces más que los ejercicios de impulso vertical (Belli, Kyröläinen \& Komi, 2002; Brughelli, Cronin \& Chaouachi, 2011; Contreras, 2009a, 2010b). Además, posee una gran transferencia a otros ejercicios y su carácter multiarticular le otorga una gran progresión, de forma opcional se puede introducir un fitball para una mayor activación de la musculatura que estabiliza el tronco (CORE) debido a la inestabilidad que otorga el material. Siguiendo las indicaciones de Contreras (2009a, 2009b, 2010b), se realizaron 2 series de 6 a 14 repeticiones. 
Tabla 2. Protocolo preventivo contextualizado aplicado en jóvenes futbolistas.

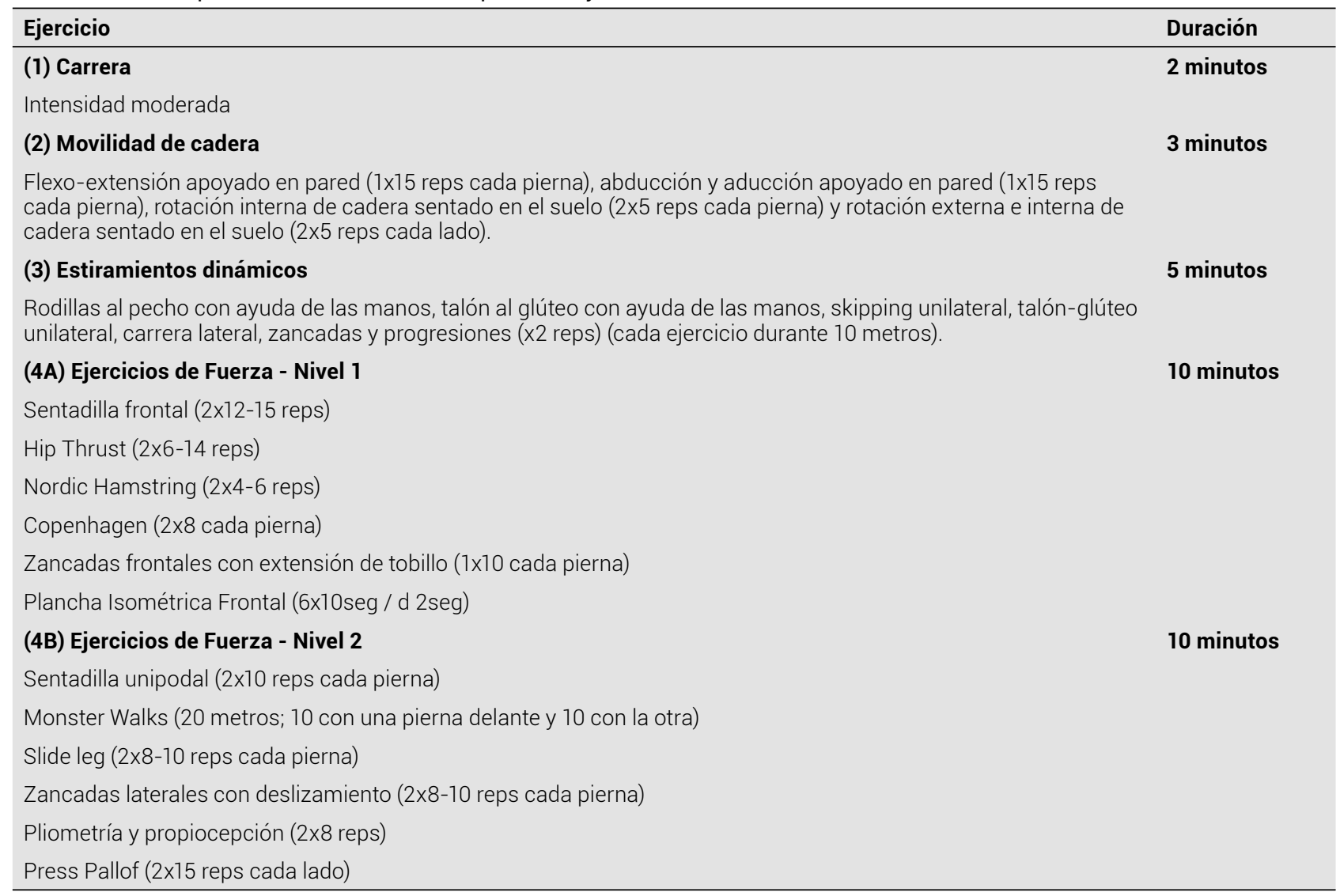

reps: repeticiones; seg: segundos; d: tiempo de descanso entre repeticiones.

Dada la gran relevancia que tiene la musculatura isquiosural sobre el rendimiento deportivo, se seleccionó el "Nordic Hamstring", un ejercicio de realización en parejas focalizado en el trabajo excéntrico de dicha musculatura. Se ha observado que este ejercicio reduce el número de lesiones y produce mejoras en fuerza muscular y salto vertical (Arnason, Andersen, Holme, Engebretsen \& Bahr, 2008; Bahr, Thorborg \& EKstrand, 2015; Brooks, Fuller, Kemp \& Reddin, 2006; Clark, Bryant, Culgan \& Hartley, 2005; Gabbett, 2000; Marshall, Lovell, Knox, Brennan \& Seigler, 2015; Sayers, 2008; Tansel, Salci, Yildirim, Kocak \& Korkusuz, 2008; Van der Horst, Smits, Petersen, Goedhart \& Backx, 2015). Se realizaron 2 series de 4 a 6 repeticiones, con una ejecución controlada.

La musculatura aductora de cadera está siendo constantemente solicitada durante la práctica del fútbol, en acciones como el golpeo (Meschini \& Pasquale, 2013). De tal modo que, para mejorar su rendimiento, se seleccionó el ejercicio de "Copenhagen" donde se trabaja la musculatura aductora de forma excéntrica.
La utilización de este ejercicio aumenta significativamente los niveles de fuerza en la musculatura objetivo (Harøy et al., 2017; Ishøi et al., 2016). Se trabaja por parejas, y la carga de trabajo está compuesta por la fuerza de la gravedad y el propio peso corporal. Hay que hacer hincapié en la importancia de la correcta sujeción por parte del compañero que no realiza el ejercicio, pues puede derivar a molestias e incluso lesiones. Se realizaron 2 series de 8 repeticiones con cada pierna.

Las zancadas frontales son un ejercicio utilizado en diferentes protocolos preventivos ya que tienen un patrón de movimiento combinado, vertical y horizontal, lo que activa una mayor musculatura, además representa una iniciación a los ejercicios monopodales (Contreras, 2009b). Como elemento diferenciador, se incluyó al final del movimiento una flexión plantar del tobillo buscando la activación del tríceps sural. Se realizó una serie de 10 zancadas por pierna.

El fortalecimiento del CORE mejora el rendimiento deportivo y ayuda a reducir la ratio lesional (Hernández-Pardo, 2015), de tal modo 
que, se incluye en el protocolo una plancha isométrica frontal con cuatro apoyos (Bliss \& Teeple, 2005). Se realizaron 6 repeticiones de 10 segundos en dicha posición, con descansos de 2 segundos entre repeticiones.

Por otro lado, el nivel 2 de dificultad de los ejercicios de fuerza quedó compuesto por 6 ejercicios: Sentadilla unipodal (2x10 reps cada pierna), Monster Walks (20 metros; 10 con una pierna delante y 10 con la otra), Slide leg (2x8-10 reps cada pierna), Zancadas laterales con deslizamiento (2x8-10 reps cada pierna), Pliometría y propiocepción ( $2 \times 8$ reps) y Press Pallof (2x15 reps cada lado). El objetivo de este nivel de dificultad es experimentar un aumento en cuanto a la activación de la musculatura, una mayor transferencia, un aumento del volumen total de entrenamiento y la reducción del número de apoyos, lo que desencadena un aumento de la dificultad de ejecución del ejercicio de forma general.

En primer lugar, se seleccionó la sentadilla monopodal, este ejercicio ha sido elegido debido a la gran activación que presenta la musculatura que estabiliza la rodilla, al aumento del grado de propiocepción y a una mayor transferencia al juego real pues la gran mayoría de los impulsos y las recepciones de los saltos se realizan con una sola pierna (Begalle, Distefano, Blackburn \& Padua, 2012; Lorenzo, 2017; Puga, 2017). Se realizaron 2 series de 10 repeticiones con cada pierna.

El "Monster Walks" tiene por objetivo la activación del glúteo medio. Este músculo posee una gran importancia pues estabiliza la rodilla y realiza la abducción de cadera, de gran trascendencia en el fútbol (Andersen et al., 2006; Distefano, Blackburn, Marshall \& Padua, 2009; Fischer \& Houtz, 1968; Krause et al., 2009; O'Sullivan, Smith \& Sainsbury, 2013; Youdas et al., 2013; Youdas, Loder, Moldenhauer, Paulsen \& Hollman, 2006). En este ejercicio se coloca una banda elástica de resistencia en los pies, tobillos o rodillas y se realiza un desplazamiento lateral mediante pequeños pasos laterales de modo que la banda elástica sirva de oposición al avance. Se realizaron 20 metros de ejercicio, 10 metros con una pierna y 10 metros con la otra.

Para una mayor activación de la musculatura isquiosural se seleccionó el "Slide Leg". Este ejercicio presenta unos altos valores de activación de toda la musculatura posterior, además, al realizarse de forma monopodal se activan significativamente los estabilizadores de cadera, rodilla y el CORE (Tsaklis et al., 2015; Yoo, 2016). Se realizaron 2 series de 8 a 10 repeticiones con cada pierna. Siguiendo con la progresión, las zancadas laterales con deslizamiento se integraron para trabajar la musculatura aductora y abductora, en este ejercicio se combina la fuerza individual de cada pierna, la flexibilidad dinámica y la inestabilidad que otorgan una gran activación de dicha musculatura (Boyle, 2017). Se realizaron 2 series de 8 a 10 repeticiones con cada pierna.

La pliometría supone niveles de activación muscular muy similares a los demandados durante la práctica del fútbol (Cometti, 2007), además de presentar grandes beneficios para los jóvenes como el aumento de la función neuromuscular (Behm, Faigenbaum, Falk \& Klentrou, 2008; Lloyd, Meyers \& Oliver, 2011) y aumento de la densidad mineral ósea (Witzke \& Snow, 2000), reduciendo el riesgo de lesiones deportivas (Markovic \& Mikulic, 2010); combinándola con un correcto trabajo propioceptivo se pueden reducir significativamente el número de lesiones (Junge, Rösch, Peterson, Graf-Baumann \& Dvorak, 2002; Olsen, Myklebust, Engebretsen, Holme \& Bahr, 2005; Sixto, 2013). Es por esto por lo que se integra un ejercicio pliométrico con elementos propioceptivos específicos. Siguiendo las indicaciones de Bedoya, Miltenberger \& Lopez (2015), se realizaron 2 series de 8 repeticiones.

Para finalizar el segundo nivel de dificultad de los ejercicios de fuerza, el press Pallof fue el ejercicio seleccionado para trabajar el CORE ya que integra un componente antirrotacional, una gran transferencia al juego real y una buena activación de la musculatura oblicua al tener que resistir una fuerza rotación, incrementada cuando los brazos se extienden frente al cuerpo (Contreras \& Schoenfeld, 2011; Gottschall, Mills \& Hastings, 2013). Se realizaron 2 series de 15 repeticiones por cada lado.

\section{Registro de lesiones}

Se registraron las lesiones tanto de entrenamientos como de partidos. Las lesiones sufridas por los porteros no fueron registradas 
puesto que realizaban un programa preventivo específico al margen del resto del equipo. La hoja de registro utilizada recoge información sobre la fecha de la lesión, la fecha de alta, el lugar y el momento en el que se produjo la lesión, el mecanismo lesional y el segmento corporal afectado, la lateralidad y la reincidencia lesiva (Fuller et al., 2006). Las lesiones fueron registradas por el fisioterapeuta de las bases del Fútbol Club Cartagena.

\section{Análisis estadístico}

Se realizó un análisis descriptivo de cada una de las variables demográficas que incluía la media y su correspondiente desviación típica. El análisis de las lesiones acontecidas a lo largo de ambas temporadas deportivas se llevó a cabo por medio de frecuencias y porcentajes. Las ratios de lesiones fueron calculadas a través de la siguiente fórmula: $n^{\circ}$ de lesiones/1000horas de exposición al deporte. Todo el análisis estadístico fue desarrollado mediante el software Microsoft Excel (2016).

\section{Resultados}

En la tabla 3 se observa el registro de lesiones durante la intervención del programa preventivo.

El análisis descriptivo ha encontrado una disminución notable del número de lesiones tras el efecto de una temporada (65\%). La variable momento exposición (entrenamiento vs competición) no ha sufrido diferencias descriptivas entre temporadas. La variable gravedad registra diferencias descriptivas en las lesiones graves (mejora del 100\%) y menores (-66,5\%) entre temporadas. La variable lateralidad ha sufrido diferencias descriptivas en la extremidad inferior no dominante (-27\%) entre temporadas.

\section{Discusión}

El principal objetivo del presente estudio fue diseñar un programa preventivo contextualizado en un equipo de jóvenes deportistas, para paliar la gran ratio lesional registrada en el fútbol (Arnason et al., 2004; Ekstrand et al., 2004; Hägglund et al., 2003; Hootman et al., 2007;
Morgan \& Oberlander, 2001; Pérez-Pemán \& Casajús-Mallén, 2013; Waldén et al., 2005; Yoon et al., 2004) derivada de sus exigencias físicas, técnicas, tácticas, psicológicas y fisiológicas (Slimani et al., 2017)

Realizando una búsqueda exhaustiva de los protocolos de prevención de lesiones aplicadas al fútbol en jóvenes deportistas, se observó que cada protocolo se divide en unos contenidos diferentes a pesar de que hay algunos que guardan una estrecha relación. El protocolo "FIFA 11+" se divide en los siguientes contenidos: (1) ejercicios de carrera, (2) ejercicios de fuerza, pliometría y equilibrio (con 3 niveles de progresión), y (3) ejercicios de carrera. Con una duración de su completa implementación de 20 minutos (Soligard et al., 2008).

Hägglund, Waldén \& Atroshi (2009) diseñan el programa neuromuscular Knäkontroll donde cada uno de los contenidos que engloba se subdividen en 4 niveles en función de la dificultad, volumen e intensidad del ejercicio: (1) sentadilla unipodal, (2) puente lumbar dinámico, (3) sentadilla bipodal, (4) plancha frontal, (5) zancadas, y (6) salto y caída. Con una duración de su completa implementación de 20 minutos.

Kiani et al. (2010) desarrollan un programa preventivo, Harmoknee, basado en 5 bloques de contenido: (1) calentamiento, (2) activación muscular, (3) equilibrio, (4) fuerza, y (5) estabilidad del CORE. La duración total de su implementación es de 20-25 minutos.

El protocolo KIPP se divide en contenidos a realizar en función de la sesión semanal en la que se realice: (1) carrera, (2) movilidad dinámica, (3) fortalecimiento, (4) pliometría, y (5) agilidad. La duración varía en función del tipo de sesión (LaBella et al., 2011).

El protocolo de prevención de lesiones contextualizado del presente trabajo está dividido en diversos contenidos; (1) carrera, (2) movilidad de cadera, (3) estiramientos dinámicos, $(4 A)$ ejercicios de fuerza - nivel 1 , y (4B) ejercicios de fuerza - nivel 2. Con una duración de su completa implementación de 20 minutos. Engloba diferentes bloques de contenidos de varios protocolos preventivos publicados por las entidades deportivas más importantes, aunándolo con las necesidades contextualizadas de la muestra. 
Tabla 3. Datos registrados durante la intervención del programa preventivo de lesiones contextualizado

\begin{tabular}{|c|c|c|c|c|}
\hline & & Temporada 2016-17 & Temporada 2017-18 & Porcentaje de mejora (\%) \\
\hline $\mathrm{N}^{0}$ lesiones & & 34 & 12 & $65 \%$ \\
\hline \multirow[t]{2}{*}{ Lugar de lesión } & Competición & $60 \%$ & $66 \%$ & $-10 \%$ \\
\hline & Entrenamiento & $40 \%$ & $33 \%$ & $17,5 \%$ \\
\hline \multirow[t]{4}{*}{ Gravedad } & Ligera & $10 \%$ & $8,3 \%$ & $17 \%$ \\
\hline & Menor & $20 \%$ & $33,3 \%$ & $-66,5 \%$ \\
\hline & Moderada & $60 \%$ & $58,3 \%$ & $2,8 \%$ \\
\hline & Grave & $10 \%$ & - & $100 \%$ \\
\hline \multirow[t]{3}{*}{ Lateralidad } & Dominante & $65 \%$ & $58 \%$ & $10,8 \%$ \\
\hline & No Dominante & $26 \%$ & $33 \%$ & $-27 \%$ \\
\hline & No aplicable & $9 \%$ & $9 \%$ & - \\
\hline
\end{tabular}

Ligera: 1 a 3 días de baja; Menor: 4 a 7 días de baja; Moderada: 8 a 28 días de baja; Grave: baja superior a 28 días.

Un objetivo secundario fue analizar los efectos del programa preventivo contextualizado sobre la incidencia lesional.

La ratio de lesiones experimentado por los jugadores durante la temporada 2016-2017 fue de 2,2/1000 horas de exposición, en cambio, durante la temporada 2017-2018, donde se realizó el protocolo preventivo propuesto en el presente trabajo, la ratio fue de 0,6/1000 horas de exposición. Estos datos se encuentran bastante alejados de los resultados obtenidos por Steffen et al. (2013) donde la ratio de lesiones tras la aplicación de un protocolo fue de 3,6/1000 horas de exposición; o el obtenido por Gatterer, Ruedl, Faulhaber, Regele \& Burtscher (2012) con 3,3/1000 horas de exposición. Incluso Soligard et al. (2008) obtiene valores muy superiores tras la realización de varios programas preventivos en los diferentes grupos experimentales (2,6/1000 horas de exposición, 3,7/1000 horas de exposición y 4/1000 horas de exposición). Algunos estudios demuestran que no hay diferencias significativas entre grupos siendo la misma ratio lesional para ambos, 9,6/1000 horas de exposición (Van Bijsterveldt et al., 2012).

En el presenteestudio, tras la implementación del programa, las lesiones se redujeron un $65 \%$ entre temporadas. Comparando este resultado con los valores obtenidos por diferentes autores, se puede establecer que los datos obtenidos por el protocolo del presente estudio reducen en mayor medida la tasa lesional respecto a los protocolos que encontramos en la literatura científica (FIFA 11+, Knäkontroll, Harmoknee y KIPP) (Figura 1).
Longo et al. (2012) establecen una reducción del $44 \%$ tras la utilización del programa "FIFA $11+$ " durante 9 meses de temporada. Steffen et al. (2013) observaron una reducción del $57 \%$ de las lesiones deportivas tras una gran adhesión al programa. En cambio, Owoeye et al. (2014), registraron 130 lesiones en 104 de los 416 jugadores, estableciendo una reducción lesional general del $41 \%$, aunque en extremidades inferiores asciende al 48\%. Este porcentaje se aproxima mucho al obtenido por Silvers-Granelli et al. (2015) en su ensayo donde el "FIFA 11+" redujo significativamente la tasa lesional al 46\%. Soligard et al. (2008) obtienen los mayores valores, $60 \%$, en cuanto a la reducción de la ratio lesional producida por este mismo protocolo. Además de estos estudios que demuestran la eficacia de los diferentes programas, existen otros en los que no se registran diferencias significativas entre el grupo control y el grupo experimental (Hammes et al., 2015; Van Bijsterveldt et al., 2012).

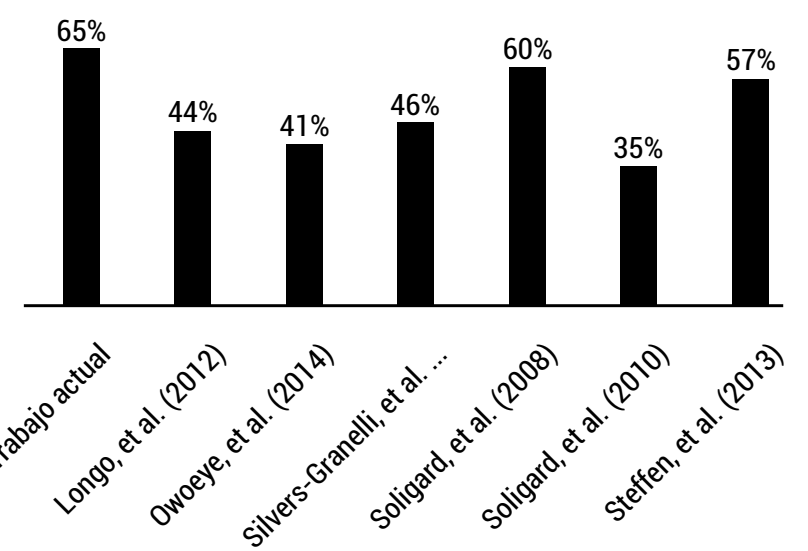

Figura 1. Comparativa de la eficacia en la reducción lesional de diferentes protocolos 
Parece ser que la incorporación de los contenidos integrados en el presente protocolo, producen una gran reducción en la ratio de lesiones. La utilización de ejercicios de movilidad de cadera, así como de estiramientos dinámicos parecen mejorar la activación muscular, el ROM, el rendimiento y la reducción lesional (Ayala \& Sainz de Baranda, 2010; Boyle, 2017; Fousekis et al., 2011; Mahieu et al., 2007; Taunton et al., 2002). Sabiendo que las lesiones relacionadas con la musculatura aductora de cadera representan del 4\% al 19\% (Pedrinelli, Da Cunha, Thiele \& Kullak, 2013) se hace imprescindible incorporar ejercicios que mejoren el rendimiento de estos músculos ("Copenhagen" y zancadas laterales con deslizamientos), a pesar de que la gran mayoría de los protocolos no les den una gran importancia y no incorporen ejercicios que mejoren su rendimiento (Harøy et al., 2017). La incorporación de ejercicios que potencien la acción de estos músculos parece reducir la ratio lesional.

A pesar de conocer la importancia del glúteo medio en la estabilización de rodilla, lo que reduce el riesgo lesional (Benito Peinado, 2008), no se encuentran protocolos en la literatura que trabajen dicha musculatura de forma específica. Este hecho puede ser uno de los aspectos que ha marcado la diferencia entre el protocolo del presente estudio y el resto. Siguiendo con la articulación de la rodilla y el tobillo, existen diversos estudios que concluyen que tener un alto grado de propiocepción es un aspecto de gran relevancia en la prevención de lesiones (Junge, Rösch, Peterson, Graf-Baumann \& Dvorak, 2002; Olsen, Myklebust, Engebretsen, Holme \& Bahr, 2005; Sixto, 2013). Analizando los diferentes programas, todos trabajan la propiocepción de forma aislada, sin transferencia real al juego, lo que reduce la eficacia de este trabajo (Smith \& Bruce-Low, 2004), al realizar una combinación entre pliometría y propiocepción, la transferencia es positiva y parece ser que se reducen las lesiones derivadas, como en el presente protocolo.

La musculatura del tríceps sural es una de las grandes olvidadas en todos los protocolos. No se han encontrado protocolos preventivos generales que trabajen dicha musculatura, a pesar de ser una de las que mayor número de lesiones leves presenta (Fuller et al., 2006; Pedrinelli et al., 2013) y una de las causantes de la modificación en la técnica de la carrera, produciendo alteraciones en los patrones motores e incrementando el riesgo de lesión de LCA (Esparza, 2007; Pedret, 2013). De tal modo que éste podría ser otro aspecto diferenciador entre el protocolo preventivo contextualizado y el resto de programas que se han encontrado en la literatura científica.

Por último, se establece que la gravedad lesional es otro aspecto a remarcar del presente protocolo, pues no se han obtenido lesiones graves en la temporada 2017-2018. Este dato podría darse debido en gran medida a la gran focalización del trabajo preventivo de la articulación de la rodilla, ya que es la zona donde se produce una de las lesiones más temidas por los futbolistas debido a su gravedad, la rotura de LCA, además de otras lesiones que engloban diferentes ligamentos y/o meniscos (Noya \& Sillero, 2012). Los valores obtenidos pueden ser debidos a que la muestra no había realizado nunca un programa preventivo durante toda la temporada. Además, los jugadores iniciaron una dieta controlada por el departamento de nutrición deportiva del Fútbol Club Cartagena, aspecto de vital relevancia para la prevención de lesiones deportivas y la correcta recuperación tras los entrenamiento y competiciones, así como una buena composición corporal (Martínez-Sainz, Urdampilleta-Otegui \& Mielgo-Ayuso, 2013).

Algunas limitaciones del presente estudio deben ser reconocidas. El tamaño de la muestra fue pequeño. No se tuvo en cuenta la posición especifica de cada jugador, pues las demandas físicas varían de una posición a otra (Noya, Gómez-Carmona, Garcia-Marco, Moliner-Urdiales \& Sillero-Quintana, 2014). Se precisa de material deportivo específico para la correcta realización del protocolo. Además, no hay que olvidar uno de los principios fundamentales del entrenamiento, la individualización, pues cada deportista responde de forma diferente ante un mismo estímulo debido a sus características personales, de tal modo que tendrá un mayor éxito la realización de protocolos preventivos individualizados atendiendo a las características y necesidades de cada jugador. 


\section{Conclusiones}

El programa preventivo diseñado presenta un enfoque integral de los protocolos publicados previamente. La contextualización está basada en las pruebas de valoración del riesgo de lesión. El programa preventivo de lesiones contextualizado presenta una disminución del riesgo de lesión del 65\%.

\section{Aplicaciones prácticas}

En el presente estudio se propone un programa preventivo para la reducción de las lesiones en jóvenes jugadores de fútbol de categoría juvenil. Dada la elevada tasa de reducción lesiva obtenida, el protocolo propuesto puede ser sujeto de nuevos estudios para comprobar su eficacia en otro tipo de muestra, así como proporcionar una herramienta de trabajo a los profesionales del deporte y de la salud que quieran reducir notablemente el número y la gravedad de lesiones.

\section{Financiación}

Este estudio es parte del Proyecto de Investigación financiado por FEDER/ Ministerio de Ciencia, Innovación y Universidades Agencia Estatal de Investigación/ Proyecto "Estudio del riesgo de lesión en jóvenes deportistas a través de redes de inteligencia artificial" (DEP2017-88775-P).

\section{Referencias}

Acero, R. M., \& Peñas, C. L. (2005). Análisis de variables determinantes en el fútbol de alto rendimiento: el tiempo de posesión del balón (abriendo la caja negra del fútbol). Red: Revista de Entrenamiento Deportivo, 19(2), 13-19.

Andersen, L. L., Magnusson, S. P., Nielsen, M., Haleem, J., Poulsen, K., \& Aagaard, P. (2006). Neuromuscular activation in conventional therapeutic exercises and heavy resistance exercises: implications for rehabilitation. Physical Therapy, 86(5), 683-697. https://pubmed.ncbi.nlm.nih.gov/16649892/

Arnason, A., Andersen, T. E., Holme, I., Engebretsen, L., \& Bahr, R. (2008). Prevention of hamstring strains in elite soccer: an intervention study. Scandinavian Journal of Medicine \& Science in Sports, 18(1), 40-48 https://doi.org/10.1111/j.1600-0838.2006.00634.x

Arnason, A., Sigurdsson, S. B., Gudmundsson, A., Holme, I., Engebretsen, L., \& Bahr, R. (2004). Physical fitness, injuries, and team performance in soccer. Medicine \& Science in Sports \& Exercise, 36(2), 278-285. https://doi.org/10.1249/01.MSS.0000113478.92945.CA
Ayala, F., \& Sainz de Baranda Andújar, P. (2010). Efecto agudo del estiramiento sobre el sprint en jugadores de fútbol de división de honor juvenil. RICYDE. Revista Internacional de Ciencias del Deporte, 6(18). https://doi.org/10.5232/ricyde2010.01801

Ayala, F.,Pomares-Noguera, C., Robles-Palazón,F. J., del Pilar GarcíaVaquero, M., Ruiz-Pérez, I., Hernández-Sánchez, S., \& Croix, M. D. S. (2017). Training effects of the FIFA $11+$ and harmoknee on several neuromuscular parameters of physical performance measures. International Journal of Sports Medicine, 38(04), 278-289. https://doi.org/10.1055/s-0042-121260

Bahr, R., Thorborg, K., \& Ekstrand, J. (2015). Evidence-based hamstring injury prevention is not adopted by the majority of Champions League or Norwegian Premier League football teams: the Nordic Hamstring survey. British Journal of Sports Medicine, 49(22),1466-71. https://doi.org/10.1136/bjsports-2015-094826

Bedoya, A. A., Miltenberger, M. R., \& Lopez, R. M. (2015). Plyometric training effects on athletic performance in youth soccer athletes: a systematic review. The Journal of Strength \& Conditioning Research, 29(8), 2351-2360. https://doi.org/10.1519/jsc.0000000000000877

Begalle, R. L., Distefano, L. J., Blackburn, T., \& Padua, D. A. (2012). Quadriceps and hamstrings coactivation during common therapeutic exercises. Journal of Athletic Training, 47(4), 396405. https://doi.org/10.4085/1062-6050-47.4.01

Behm, D. G., Faigenbaum, A. D., Falk, B., \& Klentrou, P. (2008). Canadian Society for Exercise Physiology position paper resistance training in children and adolescents. Applied Physiology, Nutrition, and Metabolism, 33(3), 547-561. https://doi.org/10.1139/h08-020

Belli, A., Kyröläinen, H. \& Komi,P.V. (2002). Momentand power oflower limb joints in running. International Journal of Sports Medicine, 23(2), 136-141. https://doi.org/10.1055/s-2002-20136

Benito Peinado, P. J. (2008). Conceptos básicos del entrenamiento con cargas: de la musculación al wellness. España: Editorial Kinesis.

Bliss, L. S., \& Teeple, P. (2005). Core stability: the centerpiece of any training program. Current Sports Medicine Reports, 4(3), 179183. https://doi.org/10.1007/s11932-005-0064-y

Boeckh-Behrens, W. U. \& Buskies, W. (2005). Entrenamiento de la fuerza. Barcelona, España: Paidotribo.

Bompa, T. O. (2000). Periodización del entrenamiento deportivo. Barcelona: Paidotribo.

Boyle, M. (2017). El entrenamiento funcional aplicado a los deportes. Madrid, España: Ediciones Tutor, S.A.

Brandt, D. J. F. (2017). Análisis Estadístico de Lesiones en Fútbol Juvenil-Asociación Argentina de Traumatología del Deporte. Revista de la Asociación Argentina de Traumatología del Deporte, 24(1). https://g-se.com/analisis-estadistico-delesiones-en-futbol-juvenil-2350-sa-U5a2181 cdc2068

Brooks, J. H., Fuller, C. W., Kemp, S. P., \& Reddin, D. B. (2006). Incidence, risk, and prevention of hamstring muscle injuries in professional rugby union. The American Journal of Sports Medicine, 34(8), 1297-1306. https://doi.org/10.1177/0363546505286022

Brughelli, M., Cronin, J. \& Chaouachi, A. (2011). Effects of running velocity on running kinetics and kinematics. The Journal of Strength \& Conditioning Research, 25(4), 933-939. https://doi.org/10.1519/jsc.0b013e3181c64308

Clark, R., Bryant, A., Culgan, J. P., \& Hartley, B. (2005). The effects of eccentric hamstring strength training on dynamic jumping performance and isokinetic strength parameters: a pilot study on the implications for the prevention of hamstring injuries. Physical Therapy in Sport, 6(2), 67-73. https://doi.org/10.1016/j.ptsp.2005.02.003

Cometti, G. (2007). La preparación física en el fútbol. Barcelona, España: Paidotribo.

Contreras, B. (2009a). Dispelling the Glute Myth. Obtenido de sitio web de T Nation: http://www.t-nation.com/free_online_article/ sports_body_training_performance/dispelling_the_glute_myth 
Contreras, B. (2009b). Advanced Glute Training. Obtenido de sitio web de T Nation: https://www.t-nation.com/training/ advanced-glute-training

Contreras, B. (2010a). Inside the Muscles: Best Legs, glute and calf exercise. Obtenido de sitio web de T Nation: https://www.tnation.com/training/inside-the-muscles-best-leg-glute-andcalf-exercises/

Contreras, B. (2010b). The top five glute exercise. CW Trainning Systems, LLC and Charlie Weingroff

Contreras, B., \& Schoenfeld, B. (2011). To crunch or not to crunch: An evidence-based examination of spinal flexion exercises, their potential risks, and their applicability to program design. Strength \& Conditioning Journal, 33(4), 8-18. http://dx.doi.org/10.1519/SSC.0b013e3182259d05

Cross, M., Williams, S., Kemp, S. P., Fuller, C., Taylor, A., Brooks, J., ... \& Stokes, K. (2018). Does the reliability of reporting in injury surveillance studies depend on injury definition? Orthopaedic Journal of Sports Medicine, 6(3), 2325967118760536 https://doi.org/10.1177/2325967118760536

Distefano, L. J., Blackburn, J. T., Marshall, S. W., \& Padua, D. A. (2009). Gluteal muscle activation during common therapeutic exercises. Journal of Orthopaedic \& Sports Physical Therapy, 39(7), 532-540. https://doi.org/10.2519/jospt.2009.2796

Drawer, S., \& Fuller, C. W. (2002). Evaluating the level of injury in English professional football using a risk based assessment process. British journal of sports medicine, 36(6), 446-451. https://doi.org/10.1136/2Fbjsm.36.6.446

Eirale, C., Gillogly, S., Singh, G., \& Chamari, K. (2017). Injury and illness epidemiology in soccer-effects of global geographical differences-a call for standardized and consistent research studies. Biology of Sport, 34(3), 249. https://doi.org/10.5114/2Fbiolsport.2017.66002

Ekstrand, J., Waldén, M., \& Hägglund, M. (2004). A congested football calendar and the wellbeing of players: correlation between match exposure of European footballers before the World Cup 2002 and their injuries and performances during the World Cup. British Journal of Sports Medicine, 38(4), 493-497. https:// doi.org/10.1136/bjsm.2003.009134

Esparza, J. (2007). Hipótesis: Causa de lesión del ligamento cruzado anterior en futbolistas. Efisioterapia, 3. https:// www.efisioterapia.net/articulos/hipotesis-causa-lesion-delligamento-cruzado-anterior-futbolistas

Fischer, F. J., \& Houtz, S. J. (1968). Evaluation of the function of the gluteus maximus muscle. An electromyographic study. American Journal of Physical Medicine \& Rehabilitation, 47(4), 182-191.

Fousekis, K., Tsepis, E., Poulmedis, P., Athanasopoulos, S., \& Vagenas, G. (2011). Intrinsic risk factors of non-contact quadriceps and hamstring strains in soccer: a prospective study of 100 professional players. British Journal of Sports Medicine, 45(9), 709-714. https://doi.org/10.1136/bjsm.2010.077560

Fuller, C. W., Ekstrand, J., Junge, A., Andersen, T. E., Bahr, R., Dvorak, J., ... \& Meeuwisse, W. H. (2006). Consensus statement on injury definitions and data collection procedures in studies of football (soccer) injuries. Scandinavian Journal of Medicine \& Science in Sports, 16(2), 83-92.

https://doi.org/10.1136/2Fbjsm.2005.025270

Gabbett, T. J. (2000). Incidence, site, and nature of injuries in amateur rugby league over three consecutive seasons. British Journal of Sports Medicine, 34(2), 98-103. https://doi. org/10.1136/2Fbjsm.34.2.98

Gatterer, H., Ruedl, G., Faulhaber, M., Regele, M., \& Burtscher, M. (2012). Effects of the performance level and the FIFA" 11 " injury prevention program on the injury rate in Italian male amateur soccer players. The Journal of Sports Medicine and Physical Fitness, 52(1), 80-84.

Gottschall, J. S., Mills, J., \& Hastings, B. (2013). Integration core exercises elicit greater muscle activation than isolation exercises. The Journal of Strength \& Conditioning Research, 27(3), 590-596.

https://doi.org/10.1519/jsc.0b013e31825c2cc7
Gribble, P. A., Terada, M., Beard, M. Q., Kosik, K. B., Lepley, A. S., McCann, R. S., Pietrosimone, B.G., \& Thomas, A. C. (2016). Prediction of lateral ankle sprains in football players based on clinical tests and body mass index. The American Journal of Sports Medicine, 44(2), 460-467. https://doi.org/10.1177/0363546515614585

Hachana, Y., Chaabène, H., Nabli, M. A., Attia, A., Moualhi, J., Farhat, N., \& Elloumi, M. (2013). Test-retest reliability, criterion-related validity, and minimal detectable change of the Illinois agility test in male team sport athletes. The Journal of Strength \& Conditioning Research, 27(10), 2752-2759. https://doi.org/10.1519/jsc.0b013e3182890ac3

Hägglund, M., Waldén, M., \& Atroshi, I. (2009). Preventing knee injuries in adolescent female football players - design of a cluster randomized controlled trial. BMC Musculoskeletal Disorders, 10, 75. http://doi.org/10.1186/1471-2474-10-75

Hägglund, M., Waldén, M., \& Ekstrand, J. (2003). Exposure and injury risk in Swedish elite football: a comparison between seasons 1982 and 2001. Scandinavian Journal of Medicine \& Science in Sports, 13(6), 364-370. https://doi.org/10.1046/j.1600-0838.2003.00327.x

Hammes, D., Aus der Fünten, K., Kaiser, S., Frisen, E., Bizzini, M. \& Meyer, T. (2015). Injury prevention in male veteran football players-a randomised controlled trial using "FIFA 11t". Journal of Sports Sciences, 33(9), 873-881. https://doi.org/10.1080/02640414.2014.975736

Harøy, J., Thorborg, K., Serner, A., Bjørkheim, A., Rolstad, L. E., Hölmich, P., ... \& Andersen, T. E. (2017). Including the copenhagen adduction exercise in the FIFA 11+ provides missing eccentric hip adduction strength effect in male soccer players: A randomized controlled trial. The American Journal of Sports Medicine, 45(13), 3052-3059. https://doi.org/10.1177/0363546517720194

Haynes, T., Bishop, C., Antrobus, M., \& Brazier, J. (2018). The validity and reliability of the My jump 2 app for measuring the reactive strength index and drop jump performance. The Journal of Sports Medicine and Physical Fitness, 59(2), 253-258. https://doi.org/10.23736/S0022-4707.18.08195-1

Hernández-Pardo, A. (2015). Efectos del fortalecimiento del tronco sobre la prevención de lesiones (Tesis de pregrado) Elche, España: Universidad Miguel Hernández.

Hootman, J. M., Dick, R., \& Agel, J. (2007). Epidemiology of collegiate injuries for 15 sports: summary and recommendations for injury prevention initiatives. Journal of Athletic Training, 42(2), 311.

Ishøi, L., Sørensen, C. N., Kaae, N. M., Jørgensen, L. B., Hölmich, P., \& Serner, A. (2016). Large eccentric strength increase using the Copenhagen Adduction exercise in football: A randomized controlled trial. Scandinavian Journal of Medicine \& Science in Sports, 26(11), 1334-1342. https://doi.org/10.1111/sms. 12585

Junge, A., Rösch, D., Peterson, L., Graf-Baumann, T., \& Dvorak, J. (2002). Prevention of soccer injuries: a prospective intervention study in youth amateur players. The American Journal of Sports Medicine, 30(5), 652-659. https://doi.org/10.1177/03635465020300050401

Kerr, Z. Y., Kroshus, E., Grant, J., Parsons, J. T., Folger, D., Hayden, R., \& Dompier, T. P. (2016). Epidemiology of National Collegiate Athletic Association Men's and Women's Cross-Country Injuries, 2009-2010 through 2013-2014. Journal of Athletic Training, 51(1), 57-64. https://doi.org/10.4085/1062-6050-51.1.10

Kiani, A., Hellquist, E., Ahlqvist, K., Gedeborg, R., \& Byberg, L. (2010). Prevention of soccer-related knee injuries in teenaged girls. Archives of Internal Medicine, 170(1), 43-49. https://doi.org/10.1001/archinternmed.2009.289

Krause, D. A., Jacobs, R. S., Pilger, K. E., Sather, B. R., Sibunka, S P., \& Hollman, J. H. (2009). Electromyographic analysis of the gluteus medius in five weight-bearing exercises. The Journal of Strength \& Conditioning Research, 23(9), 2689-2694. https://doi.org/10.1519/jsc.0b013e3181bbe86 
LaBella, C. R., Huxford, M. R., Grissom, J., Kim, K. Y., Peng, J., \& Christoffel, K. K. (2011). Effect of neuromuscular warm-up on injuries in female soccer and basketball athletes in urban public high schools: cluster randomized controlled trial. Archives of Pediatrics \& Adolescent Medicine, 165(11), 10331040. https://doi.org/10.1001/archpediatrics.2011.168

Larruskain, J., Lekue, J. A., Diaz, N., Odriozola, A., \& Gil, S. M. (2018). A comparison of injuries in elite male and female football players: A five-season prospective study. Scandinavian Journal of Medicine \& Science in Sports, 28(1), 237-245. https://doi.org/10.1111/sms.12860

Llana-Belloch, S., Pérez-Soriano, P., \& Lledó-Figueres, E. (2010). La epidemiología en el fútbol: una revisión sistemática. Revista Internacional de Medicina y Ciencias de la Actividad Física y del Deporte, 10(37). http://cdeporte.rediris.es/revista/revista37/ artfutbol130.htm

Lloyd, R. S., Meyers, R. W. \& Oliver, J. L. (2011). The natural development and trainability of plyometric ability during childhood. Strength \& Conditioning Journal, 33(2), 23-32. https://doi.org/10.1519/SSC.0b013e3182093a27

Logerstedt, D., Grindem, H., Lynch, A., Eitzen, I., Engebretsen, L., Risberg, M. A., ... \& Snyder-Mackler, L. (2012). Single-legged hop tests as predictors of self-reported knee function after anterior cruciate ligament reconstruction: the Delaware-Oslo ACL cohort study. The American Journal of Sports Medicine, 40(10), 2348-2356. https://doi.org/10.1177/0363546512457551

Longo, U. G., Loppini, M., Berton, A., Marinozzi, A., Maffulli, N., \& Denaro, V. (2012). TheFIFA 11 + program is effective in preventing injuries in elite male basketball players: a cluster randomized controlled trial. The American Journal of Sports Medicine, 40(5), 996-1005. https://doi.org/10.1177/0363546512438761

Lorenzo, A. (2017). Proceso de readaptación y reentrenamiento tras una lesión condral rotuliana. (Tesis de pregrado) Elche, España: Universidad Miguel Hernández.

Mahieu, N. N., McNair, P., De Muynck, M., Stevens, V., Blanckaert, I., Smits, N., \& Witvrouw, E. (2007). Effect of static and ballistic stretching on the muscletendon tissue properties. Medicine and Science in Sports and Exercise, 39(3), 494-501. https://doi.org/10.1249/01.mss.0000247004.40212.f7

Markovic, G., \& Mikulic, P. (2010). Neuro-musculoskeletal and performance adaptations to lower-extremity plyometric training. Sports Medicine, 40(10), 859-895 https://doi.org/10.2165/11318370-000000000-00000

Marshall, P. W., Lovell, R., Knox, M. F., Brennan, S. L., \& Siegler, J. C. (2015). Hamstring fatigue and muscle activation changes during six sets of Nordic hamstring exercise in amateur soccer players. The Journal of Strength \& Conditioning Research, 29(11), 3124-3133. https://doi.org/10.1519/jsc.0000000000000966

Martínez-Sanz, J. M., Urdampilleta-Otegui, A., \& Mielgo-Ayuso, J. (2013). Necesidades energéticas, hídricas y nutricionales en el deporte. Motricidad. European Journal of Human Movement, $30,37-52$ https: / / dialnet.unirioja.es/servlet/articulo?codigo $=4775529$

Meschini, N. S., \& Pasquale, F. (2013). Aspectos anatómicosfuncionales del remate al arco con el empeine en el fútbol. $X$ Congreso Argentino y V Latinoamericano de Educación Física y Ciencias. La Plata: Universidad Nacional de La Plata. Facultad de Humanidades y Ciencias de la Educación. Departamento de Educación Física. http://sedici.unlp.edu.ar/handle/10915/38781

Morgan, B. E., \& Oberlander, M. A. (2001). An examination of injuries in major league soccer: the inaugural season. The American Journal of Sports Medicine, 29(4), 426-430. https://doi.org/10.1177/03635465010290040701

Mufty, S., Bollars, P., Vanlommel, L., Van Crombrugge, K., Corten, K., \& Bellemans, J. (2015). Injuries in male versus female soccer players: epidemiology of a nationwide study. Acta Orthopaedica Belgica, 81 (2), 289-295. http://actaorthopaedica. be/assets/2307/18-Mufty_et_al.pdf
Negra, Y., Chaabene, H., Amara, S., Jaric, S., Hammami, M., \& Hachana, Y. (2017). Evaluation of the Illinois Change of Direction Test in Youth Elite Soccer Players of Different Age. Journal of Human Kinetics, 58(1), 215-224. https://doi.org/10.1515/2Fhukin-2017-0079

Noya, J., \& Sillero, M. (2012). Incidence of injuries in Spanish professional football over a season: Days off due to injury. Apunts Medicina de I" Esport (English Edition), 47(176), 115123. https://doi.org/10.1016/j.apunts.2011.10.001

Noya, J., Gómez-Carmona, P. M., Gracia-Marco, L., MolinerUrdiales, D., \& Sillero-Quintana, M. (2014). Epidemiology of injuries in First Division Spanish football. Journal of Sports Sciences, 32(13), 1263-1270. https://doi.org/10.1080/02640414.2014.884720

Noyes, F. R., Barber, S. D., \& Mangine, R. E. (1991). Abnormal lower limb symmetry determined by function hop tests after anterior cruciate ligament rupture. The American Journal of Sports Medicine, 19(5), 513-518. https://doi.org/10.1177/036354659101900518

Olsen, O. E., Myklebust, G., Engebretsen, L., Holme, I., \& Bahr, R. (2005). Exercises to prevent lower limb injuries in youth sports: cluster randomised controlled trial. BMJ, 330(7489), 449. https://doi.org/10.1136/bmi.38330.632801.8f

Orchard, J. W., Waldén, M., Hägglund, M., Orchard, J. J., Chivers, I., Seward, H., \& Ekstrand, J. (2013). Comparison of injury incidences between football teams playing in different climatic regions. Open Access Journal of Sports Medicine, 4, 251. https://doi.org/10.2147/2FOAJSM.S52417

O'Sullivan, K., Smith, S. M., \& Sainsbury, D. (2013). Análisis Electromiográfico de las Tres Subdivisiones del Glúteo Medio Durante Ejercicios con Soporte de Peso Corporal-Equipo Physical. PublicE. https://g-se.com/ analisis-electromiografico-de-las-tres-subdivisionesdel-gluteo-medio-durante-ejercicios-con-soporte-de-pesocorporal-1556-sa-G57cfb27228a9c

Owoeye, O. B., Akinbo, S. R., Tella, B. A., \& Olawale, O. A. (2014). Efficacy of the FIFA 11+ warm-up programme in male youth football: a cluster randomised controlled trial. Journal of Sports Science \& Medicine, 13(2), 321

Pedret, C. (2013). El músculo sóleo: anatomía, histología, topografía y pronóstico de las lesiones musculares (Tesis de doctorado). Barcelona, España: Universidad Autónoma de Barcelona.

Pedrinelli, A., Da Cunha Filho, G. A. R., Thiele, E. S., \& Kullak, O. P. (2013). Estudo epidemiológico das lesões no futebol profissional durante a Copa América de 2011, Argentina. Revista Brasileira de Ortopedia, 48(2), 131-136. https://doi.org/10.1016/j.rbo.2012.09.001

Pérez-Pemán, D., \& Casajús-Mallén, J. A. (2013). Lesiones en las extremidades inferiores en jugadoras de fútbol: revisión sistemática (Tesis de pregrado). España: Universidad de Zaragoza.

Puga, K. M. (2017). Efectividad de la aplicación de un programa de entrenamiento funcional en jugadores de fútbol amateur masculino que hayan sufrido esquince de tobillo grado I, de 18-25 años, de la Pontificia Universidad Católica del Ecuador en el periodo de noviembre del 2016 a marzo del 2017 (Tesis de pregrado). Ecuador: Pontificia Universidad Católica del Ecuador.

Reid, A., Birmingham, T. B., Stratford, P. W., Alcock, G. K., \& Giffin, J. R. (2007). Hop testing provides a reliable and valid outcome measure during rehabilitation after anterior cruciate ligament reconstruction. Physical Therapy, 87(3), 337-349. https://doi.org/10.2522/pti.20060143

Robles-Palazón, F., \& Sainz de Baranda, P. (2017). Programas de entrenamiento neuromuscular para la prevención de lesiones en jóvenes deportistas. Revisión de la literatura. SPORT TKRevista EuroAmericana de Ciencias del Deporte, 6(2), 115-126. https://doi.org/10.6018/300451

Romiti, M. A., Finch, C. F., \& Gabbe, B. J. (2007). A prospective cohort study of the incidence of injuries among junior Australian 
football players-evidence for a playing age level effect. British Journal of Sports Medicine, 42(6), 441-446. https://doi.org/10.1136/bjsm.2007.042721

Roos, K. G., Wasserman, E. B., Dalton, S. L., Gray, A., Djoko, A., Dompier, T. P., \& Kerr, Z. Y. (2017). Epidemiology of 3825 injuries sustained in six seasons of National Collegiate Athletic Association men's and women's soccer (2009/20102014/2015). British Journal of Sports Medicine, 51, 1029-1034.

Roozen, M. (2004). Illinois agility test. NSCA's Performance Training Journal, 3(5), 5-6.

Sayers, B. E. (2008). The Nordic eccentric hamstring exercise for injury prevention in soccer players. Strength \& Conditioning Journal, 30(4), 56-58. https://doi.org/10.1519/SSC.0b013e31817f985d

Silvers-Granelli, H., Mandelbaum, B., Adeniji, O., Insler, S., Bizzini, M., Pohlig, R., ... \& Dvorak, J. (2015). Efficacy of the FIFA 11+ injury prevention program in the collegiate male soccer player. The American Journal of Sports Medicine, 43(11), 2628-2637. https://doi.org/10.1177/0363546515602009

Sixto, M. (2013). Revisión Sistemática: Influencia de la propiocepción en la recuperación funcional del esguince de tobillo (Tesis de pregrado). España: Universidade da Coruña, España.

Slimani, M., Bouazizi, M., Chortane, S. G., \& Cheour, F. (2014). SocialProfessional Factors and Predominance of Burnout Syndrome in Tunisian Male Soccer Players. IOSR Journal of Research \& Method in Education (IOSR-JRME), 4(3), 31-34.

Slimani, M., Bragazzi, N. L., Znazen, H., Paravlic, A., Azaiez, F., \& Tod, D. (2017). Psychosocial predictors and psychological prevention of soccer injuries: A systematic review and metaanalysis of the literature. Physical Therapy in Sport, 32, 293300. https://doi.org/10.1016/j.ptsp.2018.05.006

Smith, D., \& Bruce-Low, S. (2004). Strength training methods and the work of Arthur Jones. Journal of Exercise Physiology, 7(6), 52-68

Soligard, T., Myklebust, G., Steffen, K., Holme, I., Silvers, H., Bizzini, M., ... \& Andersen, T. E. (2008). Comprehensive warm-up programme to prevent injuries in young female footballers: cluster randomised controlled trial. BMJ, 337, a2469. https://doi.org/10.1136/bmj.a2469

Soligard, T., Nilstad, A., Steffen, K., Myklebust, G., Holme, I., Dvorak, J., ... \& Andersen, T. E. (2010). Compliance with a comprehensive warm-up programme to prevent injuries in youth football. British Journal of Sports Medicine, 44(11), 787793. http://dx.doi.org/10.1136/bjsm.2009.070672

Stefanyshyn, D., \& Nigg, B.M. (1998). Contribution of the lower extremity joints to mechanical energy in running vertical jumps and running long jumps. Journal of Sports Sciences, 16(2), 177-186. https://doi.org/10.1080/026404198366885

Steffen, K., Emery, C.A., Romiti, M., Kang, J., Bizzini, M., Dvorak, J., ... \& Meeuwisse, W. H. (2013). High adherence to a neuromuscular injury prevention programme (FIFA 11+) improves functional balance and reduces injury risk in Canadian youth female football players: a cluster randomised trial. British Journal of Sports Medicine, 47(12), 794-802. https://doi.org/10.1136/bjsports-2012-091886

Steffen , K., Myklebust, G., Olsen, O. E., Holme, I., \& Bahr, R. (2008). Preventing injuries in female youth football-a cluster randomized controlled trial. Scandinavian Journal of Medicine \& Science in Sports, 18(5), 605-614.
Tansel, R. B., Salci, Y., Yildirim, A., Kocak, S., \& Korkusuz, F. (2008). Effects of eccentric hamstring strength training on lower extremity strength of 10-12-year-old male basketball players. Isokinetics and Exercise Science, 16(2), 81-85. https://doi.org/10.3233/IES-2008-0300

Taunton, J. E., Ryan, M. B., Clement, D. B., McKenzie, D. C., LloydSmith, D. R., \& Zumbo, B. D. (2002). A retrospective case-control analysis of 2002 running injuries. British Journal of Sports Medicine, 36(2), 95-101. https://doi.org/10.1136/bjsm.36.2.95

Tsaklis, P., Malliaropoulos, N., Mendiguchia, J., Korakakis, V., Tsapralis, K., Pyne, D., \& Malliaras, P. (2015). Muscle and intensity based hamstring exercise classification in elite female track and field athletes: implications for exercise selection during rehabilitation. Open access Journal of Sports Medicine, 6, 209. https://doi.org/10.2147/oajsm.s79189

Van Beijsterveldt, A. M., van de Port, I. G., Krist, M. R., Schmikli, S. L., Stubbe, J. H., Frederiks, J. E., \& Backx, F. J. (2012). Effectiveness of an injury prevention programme for adult male amateur soccer players: a cluster-randomised controlled trial. British Journal of Sports Medicine, 46(16), 1114-1118 https://doi.org/10.1136/bjsports-2012-091277

Van der Horst, N., Smits, D. W., Petersen, J., Goedhart, E. A., \& Backx, F. J. (2015). The preventive effect of the nordic hamstring exercise on hamstring injuries in amateur soccer players: a randomized controlled trial. The American Journal of Sports Medicine, 43(6), 1316-1323. https://doi.org/10.1177/0363546515574057

Waldén, M., Hägglund, M., \& Ekstrand, J. (2005). UEFA Champions League study: a prospective study of injuries in professional football during the 2001-2002 season. British Journal of Sports Medicine, 39(8), 542-546. https://bjsm.bmj.com/content/39/8/542

Waldén, M., Hägglund, M., Orchard, J., Kristenson, K., \& Ekstrand, J. (2013). Regional differences in injury incidence in European professional football. Scandinavian Journal of Medicine \& Science in Sports, 23(4), 424-430. https://doi.org/10.1111/j.1600-0838.2011.01409.x

Witzke, K. A., \& Snow, C. M. (2000). Effects of polymetric jump training on bone mass in adolescent girls. Medicine and Science in Sports and Exercise, 32(6), 1051-1057. https://doi.org/10.1097/00005768-200006000-00003

Yoo, W. G. (2016). Effects of bridging plus exercises with heel lift on lower extremity muscles. Journal of Physical Therapy Science, 28(5), 1582-1583. https://doi.org/10.1589/2Fjpts.28.1582

Yoon, Y. S., Chai, M., \& Shin, D. W. (2004). Football Injuries at Asian Tournaments. The American Journal of Sports Medicine, 32(1 suppl), 36-42. https://doi.org/10.1177/0095399703258781

Youdas, J. W., Foley, B. M., Kruger, B. L., Mangus, J. M., Tortorelli, A. M., Madson, T. J., \& Hollman, J. H. (2013). Electromyographic analysis of trunk and hip muscles during resisted lateral band walking. Physiotherapy Theory and Practice, 29(2), 113-123. https://doi.org/10.3109/09593985.2012.704492

Youdas, J. W., Loder, E. F., Moldenhauer, J. L., Paulsen, C. R., \& Hollman, J. H. (2006). Hip-abductor muscle performance in participants after 45 seconds of resisted sidestepping using an elastic band. Journal of Sport Rehabilitation, 15(1), 1-11. https://doi.org/10.1123/jsr.15.1.1 


\section{Anexos}

Anexo 1. Cuestionario de historial deportivo y de lesiones.

\section{DATOS PERSONALES DEL DEPORTISTA}

Nombre y Apellidos:

Fecha de nacimiento:_/ _ / _ Sexo: $\square \mathrm{M} \square \mathrm{F}$ Peso(Kg):

Email:

Teléfono:

Categoría:

Años de práctica deportiva:

Pierna dominante: Horas semanales de entrenamiento:

Indique la/s posición/es en donde te encuentras más cómodo:

Indique los clubs en los que ha tenido ficha federativa y su categoría:

\section{HISTORIAL DE LESIONES}

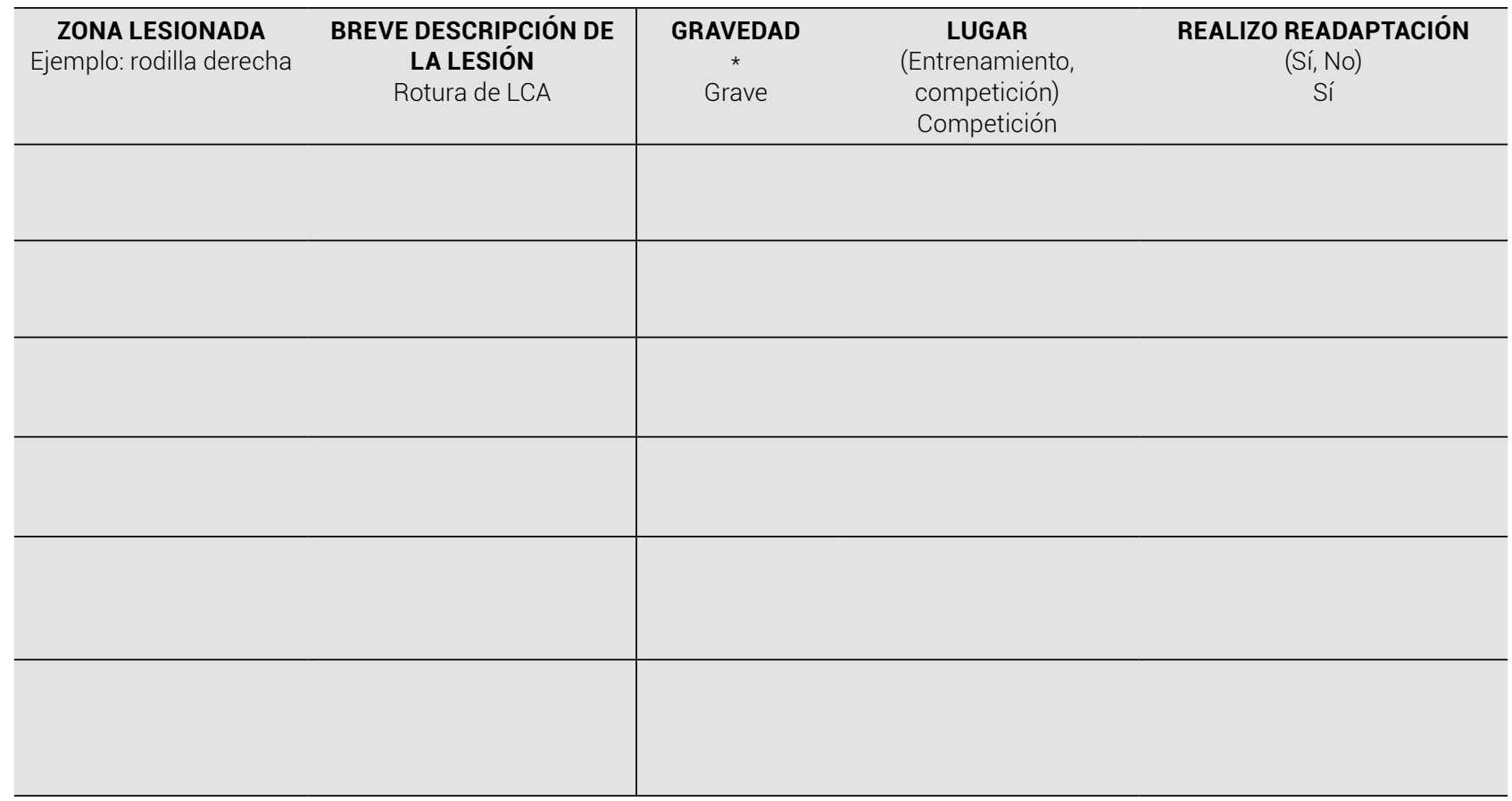

*Gravedad:

$\square$ Lesión leve ( 1 a 3 días sin participar en entrenamiento ni competiciones)

$\square$ Lesión menor (4 a 7 días sin participar en entrenamiento ni competiciones)

$\square$ Lesión moderada (8 a 28 días sin participar en entrenamiento ni competiciones)

$\square$ Lesión grave (más de 28 días sin participar en entrenamiento ni competiciones) 


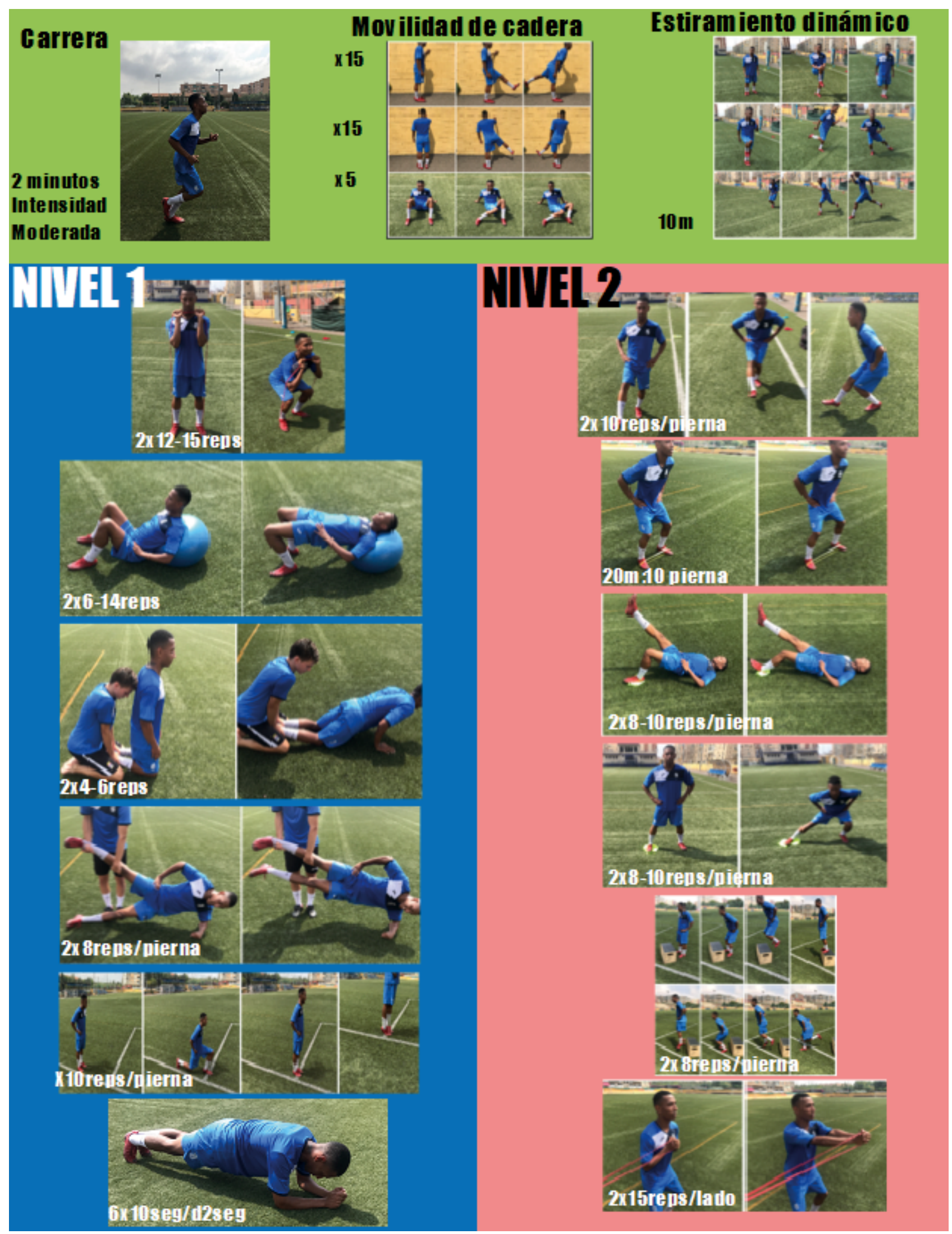

Anexo 2. Protocolo preventivo. 